\title{
Windswept deformities of the knee are challenging to manage
}

\author{
Suresh Babu * (D, Abhishek Vaish and Raju Vaishya
}

\begin{abstract}
Background: Little has been published about TKA in windswept deformities of the knees where combined varus and valgus deformities present in the same patient. Windswept deformities present with unique problems and must be addressed as two halves of a complex entity. Through this review we aim to understand the interrelation between the deformities, examine outcomes following simultaneous bilateral total knee arthroplasty in windswept deformities, and develop an algorithm for the management of windswept deformities by total knee arthroplasty.

Methods: An extensive online literature search for the keywords yielded 31 articles on which we based our review. Articles were analyzed in context to our research questions and are presented in a tabular format for quick reference and a better perspective.

Results: The abnormal biomechanics and force moment of the knee cause progressive arthritis of the knee. The valgus deformity usually precedes a varus deformity on the contralateral knee in windswept deformities. Correct restoration of mechanical tibiofemoral angles by individualizing valgus correction angles have better outcomes after TKA.

Conclusion: A well-planned and judiciously executed simultaneous bilateral total knee replacement can offer distinct advantages to the patient and surgeon and provides optimum utilization of time and resources in the management of windswept knees.
\end{abstract}

Keywords: Windswept deformities, Varus deformity, Valgus deformity, Knee, Arthroplasty, Simultaneous bilateral

\section{Introduction}

Windswept deformities (WSD) of the knee are not common presentations and pose unique challenges during total knee arthroplasty (TKA). In addition to resurfacing the arthritic surfaces of the joint, restoration of the normal biomechanics of the knee is essential [1]. WSD present a scenario with the knees at two extremes of the deformity spectrum in the coronal plane, and each extreme shows varied bony and soft tissue insufficiencies [2]. The etiopathology of the deformities is different and also needs to be addressed [3]. In a WSD, there is primarily medial compartment osteoarthritis $(\mathrm{OA})$ on the

\footnotetext{
* Correspondence: yssureshbabu@gmail.com

Department of Orthopaedics and Joint Replacement Surgery, Indraprastha Apollo Hospitals, Sarita Vihar, New Delhi 110076, India
}

side of varus deformity [2,3] and lateral compartment $\mathrm{OA}$ on the side of the valgus deformity. The soft tissues on the medial side of the knee are contracted and need to be released in a varus knee [3], whereas in a valgus knee, the soft tissues on the lateral side of the knee are contracted and require release [1-3]. There are varying degrees of patellofemoral arthritis, and patellar tracking should be optimized to obtain superior outcomes, which in many instances, especially with valgus knees, may require a lateral retinacular release [4-6]. There is a paucity of literature to help understand the influence of individual deformities on the contralateral knee and their importance in management by total knee replacement. Whether the deformities represent a continuum in the spectrum of windswept knees and what the management protocols should be are also unclear.

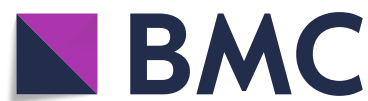

Part of Springer Nature

(c) The Author(s). 2020 Open Access This article is licensed under a Creative Commons Attribution 4.0 International License, which permits use, sharing, adaptation, distribution and reproduction in any medium or format, as long as you give appropriate credit to the original author(s) and the source, provide a link to the Creative Commons licence, and indicate if changes were made. The images or other third party material in this article are included in the article's Creative Commons licence, unless indicated otherwise in a credit line to the material. If material is not included in the article's Creative Commons licence and your intended use is not permitted by statutory regulation or exceeds the permitted use, you will need to obtain permission directly from the copyright holder. To view a copy of this licence, visit http://creativecommons.org/licenses/by/4.0/ The Creative Commons Public Domain Dedication waiver (http://creativecommons.org/publicdomain/zero/1.0/) applies to the data made available in this article, unless otherwise stated in a credit line to the data. 
We therefore reviewed the literature to identify the challenges to TKA in WSD and to contribute to an understanding of the following:

1. Whether an interrelation exists between the deformities and whether the effect of kinematics is mutually inclusive

2. The outcomes with simultaneous bilateral total knee arthroplasty (SBTKA)

3. The need for constrained designs, stem extensions, and additional procedures

4. The possibility of formulating an algorithm for total knee arthroplasty in windswept deformities

\section{Materials and methods}

We did a comprehensive literature search of the indexed databases including PubMed, ResearchGate, Google Scholar, Scopus, Medline, and Google Search using the MeSH words, "Windswept, Deformity of the knee, Total Knee Arthroplasty, Total Knee Replacement, Combined Varus-Valgus, Simultaneous bilateral total knee arthroplasty." We could find a total of 47 articles in the literature. After filtering for open access, complete text, and English language articles, we were able to reference 31 articles for our study (Fig. 1). Citations were obtained for the selected articles in the required format to create the reference section.

All the studies selected were analyzed in context to the aims of this study. We tabulated highly relevant studies and collated details of the author, journal, year of publication, level of evidence, and the conclusions drawn from the studies (Table 1). The articles were segregated and analyzed relative to biomechanics and gait, clinical significance of WSD, implications of WSD in planning for TKA, intra-operative planning of valgus correction angle (VCA), and clinical outcomes following bilateral staged or SBTKA. Adequate and appropriate referencing was done in the preparation of the manuscript. An algorithm was also prepared.

\section{Results}

Biomechanics and gait

Kutzner et al. [7] noticed a correlation between knee adduction moment and medial contact force during gait using telemetric data transmission in the instrumented knee (following TKA) and found a good correlation between external adductor moment and medial joint force. Amin et al. [8], in their study of knee adduction moment (KAM) and development of chronic knee pain in $132 \mathrm{el}-$ ders found that a greater adduction moment at the knee during activities contributes to the development of

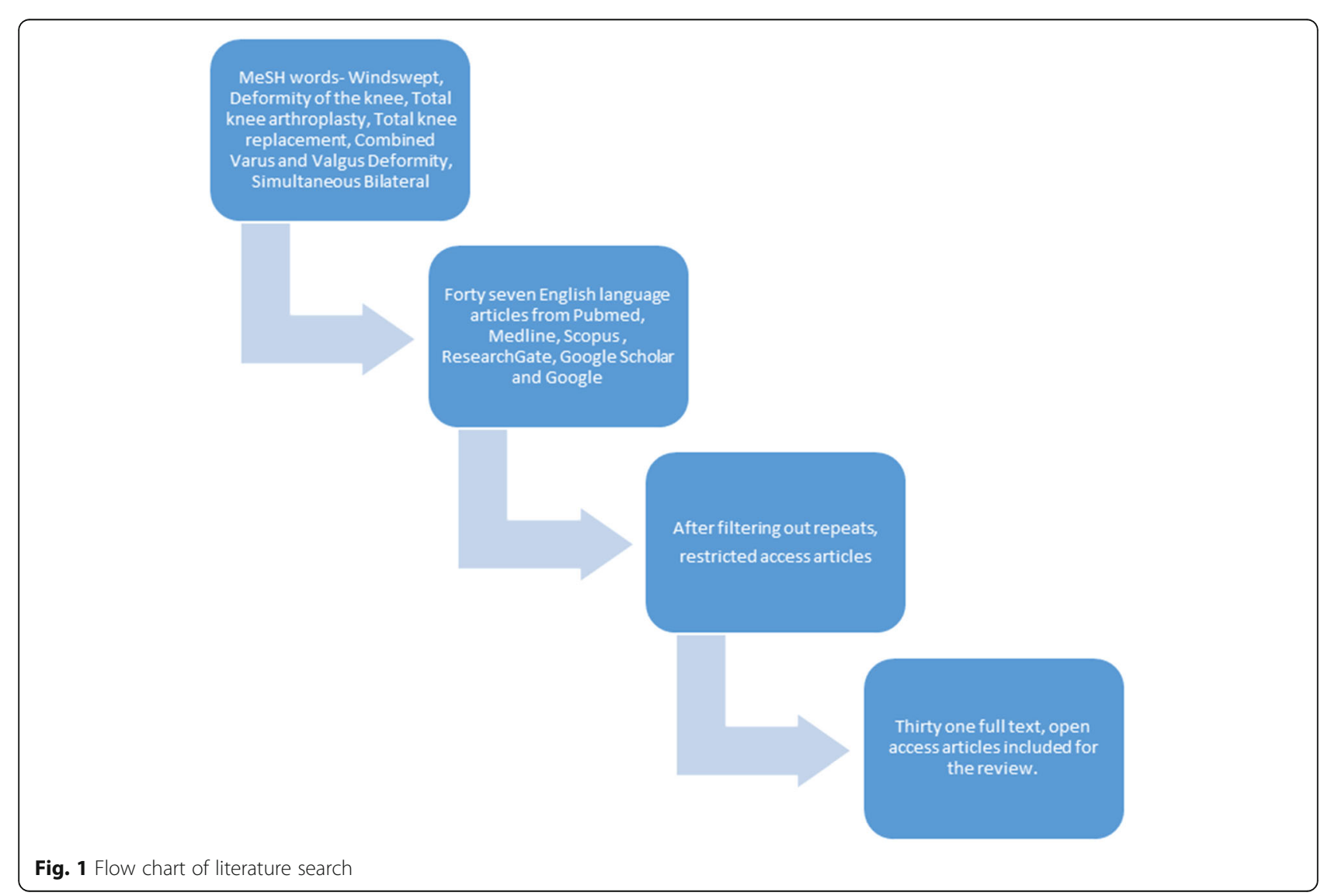


Table 1 Studies and their findings related to windswept knees

\begin{tabular}{|c|c|c|c|}
\hline & AUTHORS & YEAR & JOURNAL \\
\hline 1 & Mullaji et al & 2016 & Journal of Orthopaedics \\
\hline 2 & Howell et al & 2020 & Knee Surgery Sports Traumatology Arthros \\
\hline 3 & \multicolumn{2}{|c|}{ In-soo Song et al2008 } & Journal of Korean Knee Society \\
\hline 4 & Baldini et al & 2015 & Bone Joint Journal \\
\hline 5 & Meding et al & 2000 & The Journal of Arthroplasty \\
\hline 6 & Chapman et al & 2015 & Journal of Rheumatology \\
\hline 7 & Leena et al & 2001 & Journal of the American Medical Associatio \\
\hline 8 & Beveridge et al & 2015 & Osteoarthritis and Cartilage \\
\hline 9 & Chapman et al & 2011 & Oeteoarthritis and Cartilage \\
\hline 10 & Sugioka et al & 1990 & Orthopaedics and Traumatology \\
\hline 11 & Ganger et al & 2012 & Swiss Medical Weekly \\
\hline 12 & Case et a & & $\begin{array}{l}\text { 49th Annual Meeting of the Orthopaedic } \\
\text { Research Society }\end{array}$ \\
\hline
\end{tabular}

12 Case et al Research Society
13 Eric et al 2014 Osteoarthritis Cartilage
14 Ines Kutzner et al 2013 Plos One
15 Chapman et al 2013 The Journal of Rheumatology

16 Morrey et al 2001 Journal of Biomechanics

17 Hiyashi \& Mikawa 2004 Kawasaki Medical School

18 Rao and Reddy

2017 International Journal of Research in Orthopaed is
CONCLUSIONS

Significant difference in VCA is present between the varus and the valgus limbs in most

patients with windswept deformity undergoing TKA

Simultaneous or staged total knee arthroplasties in patients with simultaneous varus and contralateral valgus knee deformities achieved satisfactory outcomes with regard to the objective orthopedic criteria as well as the overall patient satisfaction in terms of pain relief and function.

If non-constrained components have been

used with an extensive lateral release, the adduction

may predispose

to a varus angulation (windswept deformity).

Total knee arthroplasty in patients

with windswept deformity can be expected to be successful in both knees when attention is given to proper alignment and soft tissue balancing intraoperatively

In persons with medial $\mathrm{OA}$, the contralateral knee is also at high risk of medial $\mathrm{OA}$ teral reduction in medial loading in knees by use of strategies such as lateral wedge insoles might side.

Demonstrate that in primary knee $\mathrm{OA}$

varus alignment increases risk of medial $\mathrm{OA}$ progression, that valgus alignment increases isk of lateral OA progression, that burden of malalignment predicts decline in physical

The preliminay study results do not support the hypothesis that morphological and microstructural changes in the
contralateral articular cartilage and Bone Marrow Lesions predict OA changes

Conservative management options such as lateral wedge insoles might assist in

side especially in the very early stages of the disease.

One side valgus angulation might cause a compensatory varus angulation on the contralateral side.

Poor bone quality in patients with skeletal dysplasias makes optimal correction and re-alignment

Pain in one joint

may directly and predictably affect loading in other joints.

Quite possibly other neuromuscular adaptations, together with structural

changes in cartiage and bone, account for some of the between-limb

asymmetrical loading seen in this study.

The findings that the KAM has a greater influence on femoral cartilage change and the KFM has a greater influence on tibial cartilage change provide new insight into

Good correlation between EAM and Fmed was found during the early stance phase,

In persons with medial OA, the contralateral knee is also at high risk of medial OA. Bilateral reduction in medial loading in knees by use of strategies such as lateral wedge Bilateral reduction in medial loading in knees by use of strategies such as lateral wedge
insoles might the contralateral

Subjects with $\mathrm{OA}$ compensate to reduce the knee extensor moment, and consequently the knee join loading. By analyzing a patient's gait pattern, it is judging the locomotor hatiop caused by

Patient Satisfied with the results

The mTFA may be considered one important criterion in designing treatment and planning surgery for patients with primary osteoarthritis.

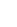


future chronic knee pain and suggested that biomechanical factors may play an important role in the pathogenesis and progression of knee pain. This finding has implications in our understanding of the development of knee osteoarthritis, which begins with abnormal alignment (deformity) and the increased force moments around the knee that lead to degenerative changes. Andriacchi et al. and Beveridge et al. [9-11] have studied the effects of gait mechanics on healthy cartilage morphology and knee OA and concluded that adduction moment during walking can be predictive of the clinical outcomes of treatment. Morrey et al. [12], in their study of knee kinematics in 139 patients with grade two knee OA, reported that subjects with OA compensate to reduce the knee extensor moment and, consequently, affect their joint loading and gait patterns. These parameters are quantitatively evaluated in measuring the locomotor handicap caused by OA. Based on these studies, this malalignment, by inference, is an essential prerequisite for the development of osteoarthritis and deformities in windswept deformities, which mutually perpetuate and aggravate osteoarthritis of the contralateral knee.

\section{Clinical significance of windswept deformities}

Sugioka et al. [13], in a retrospective study, reviewed five adult patients who had a WSD. They made an important observation that one side valgus angulation might cause a compensatory varus angulation on the contralateral side, although this finding was not substantiated by any further studies. Case et al. [14], in their presentation on the gait observational data in 53 patients, have opined that pain in one joint may directly and predictably affect loading in other joints. Rao and Reddy [15], in their study on the association of frontal plane tibiofemoral alignment with knee pain in 314 knees (195 patients), found a positive correlation between coronal tibiofemoral angles and pain in primary OA. Leena Sharma [16] observed that the burden of malalignment of the knees predicts a decline in physical function and that these effects can be detected as early as 18 months.

\section{Implications for intraoperative planning of valgus correction angle}

Mullaji et al. [17] studied the variation in femoral valgus correction angle (VCA) between the two limbs in patients with windswept deformity undergoing TKA and found that VCA in varus knees was significantly higher compared to mean VCA in the valgus knees. Shi et al. [18], in their large study on the accuracy of using individualized valgus correction angle during TKA for varus and valgus deformities of the knee, concluded that individual correction angles improve the accuracy of postoperative limb alignment after TKA compared with using fixed valgus correction guides. Nam et al. [19], in a retrospective review of 320 consecutive patients, found that the use of a variable distal femur resection angle improves femoral component alignment after TKA. Similarly, Zhou $\mathrm{K}$ et al. [20], in a radiological study, showed that individual VCA for distal femoral resection could achieve better postoperative alignment accuracy and fewer outliers of limb and femoral component malalignment in the coronal plane.

\section{Clinical outcomes following bilateral TKA in windswept deformities}

Howell et al. [21], in a prospective review of 19 patients who underwent bilateral TKA in WSD, looked into the level of implant constraint, outcome scores, and alignment after bilateral, callipered, kinematically aligned TKA and observed that no knees required semiconstrained implant or posterior cruciate ligament release, and a short tibial stem extension was used in only one valgus knee. They did not find any difference in the median postoperative "Forgotten Joint Scores" and "Oxford Knee Scores" between paired varus and valgus knees. Their management was a staged procedure of doing the knees at separated intervals and sequences, depending on the severity. They achieved one degree or less in the mean difference in postoperative distal lateral femoral angle and the proximal medial tibial angle between the varus and valgus knees using cruciateretaining implants. Song et al. [22], in their study of 14 patients of WSD who underwent SBTKA, found comparable knee scores for both deformities at an average follow-up of 18.4 months. However, Baldini et al. [23] cautioned against not using constrained components in valgus knees that needed extensive lateral release, suggesting that the adduction moment generated by the contralateral knee can predispose to varus angulation in the operated knee. Meding et al. [24] studied 20 knees with WSD undergoing bilateral TKA (simultaneous in 18 and staggered in two patients) and found the procedure to be successful in both knee groups (varus/valgus) and that proper attention to alignment and soft tissue balancing intraoperatively are essential. Hiyashi and Mikawa [25], in their case report of a 63-year-old female with WSD treated by bilateral TKA, reported a high satisfaction level in the patient following the procedure. Ganger et al. [22], in a case report of WSD in a patient with skeletal dysplasia, commented on the poor bone quality in these patients, which made optimal correction and realignment difficult.

\section{Discussion}

Windswept deformities (WSD) of the knee are so named as to describe varus deformity on one side with valgus on the contralateral side [17]; for example, if the wind 
blew across the knees from left to right, a valgus deformity would be produced on the left and a varus on the right (Fig. 2, Fig. 3). In a WSD, there is a predominantly medial compartment $\mathrm{OA}$ on the side of varus deformity and lateral compartment $\mathrm{OA}$ on the side of the valgus deformity [17, 24] (Fig. 4).

Although no etiology exists as a specific cause of windswept deformities, these deformities can be seen in inflammatory arthritides like rheumatoid arthritis, skeletal dysplasias, sequelae of childhood rickets, lateral femoral condyle dysplasia in one knee, and as a result of the overcorrection of genu varum on one side [24-26]. Malalignment at the knee increases the adduction or abduction moments around the knee and leads to deterioration of the OA changes in the knees. The burden of malalignment is proportionate to the decline in the physical function of the knee [7]. Deformities may be biomechanically interrelated going by the sequence of deformities and loading patterns, and the contralateral knee also can be safely assumed to become symptomatic in time [8-11]. Neglected deformities of the knee with WSD cause significant locomotor disability [12] and correspondingly leads to a prolonged recovery time and therefore should be addressed promptly by structured

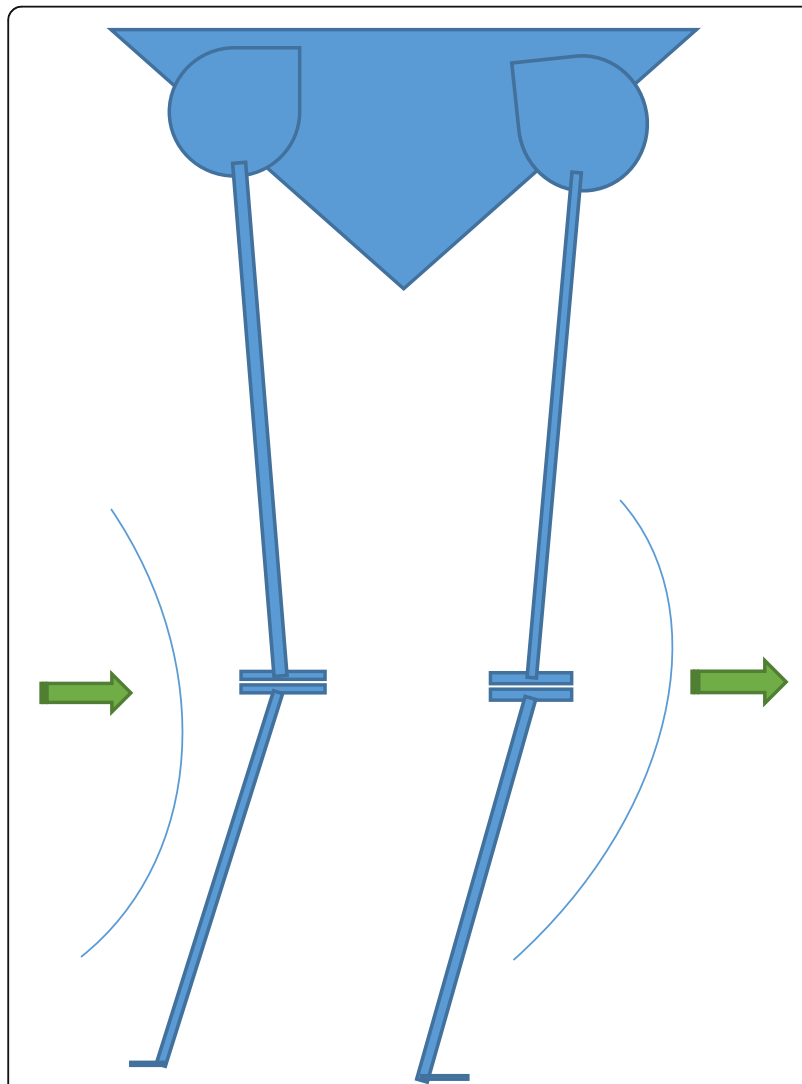

Fig. 2 Diagrammatic representation of a Windswept deformity with varus and valgus physical exercise and a muscle strengthening program postoperatively. Valgus in one knee usually proceeds a varus deformity on the contralateral knee and can be mutually compensating [13]. Abnormal loading forces in one knee due to deformity predictably affect loading patterns and loading of the contralateral knee [14]. Conditions with marked deviation in tibiofemoral alignment angles, as seen in WSD, produce considerable morbidity and abnormality in gait patterns [15]. Abnormal force moments around the knee influence progression of pain and OA of the contralateral knee [16]. Although the procedure of TKA is successful with both primary posterior stabilized and cruciate-retaining implants, the surgeon should not hesitate to increase the level of constraint and to use stem extensions if the situation so warrants [21]. Outcomes with single or staged TKA are good and comparable with TKA for bilateral knee OA without WSD [21-25]. Skeletal dysplasia, condylar hypoplasia, rheumatoid arthritis, and bony defects present both a defective and deficient bone stock during TKA, which makes the surgery challenging [26].

\section{Management}

Windswept deformities present a difficult surgical preposition in terms of $[2,3,17,24]$ of the following:

- bilateral affection

- grotesque deformities

- bone defects

- ligamentous laxity

- locomotor disability

- poor bone stock and quality as a result of dysplasia and inflammatory arthritis or sequelae of childhood rickets

- Limb length discrepancies

- peroneal nerve injury, in the valgus knee

Hence, the WSD should be approached as a single entity, and each knee should be considered as one-half of the problem. Treating the problem in its entirety makes sound clinical sense due to the following perspectives.

\section{Anatomy and biomechanical perspective}

The WSD presenting with significant malalignment and altered biomechanics with a deformity in one knee precipitating and aggravating the disease and symptoms of the contralateral knee [7] is challenging. Abnormal force moments (adductor moment in varus and abductor moment in valgus) have a cascading effect on the gait pattern, joint loading, muscle conditioning, and energy consumption [8]. The main component of the deformities is bony, with ligamentous attenuation being contributory. Therefore, the deformities in WSD are 


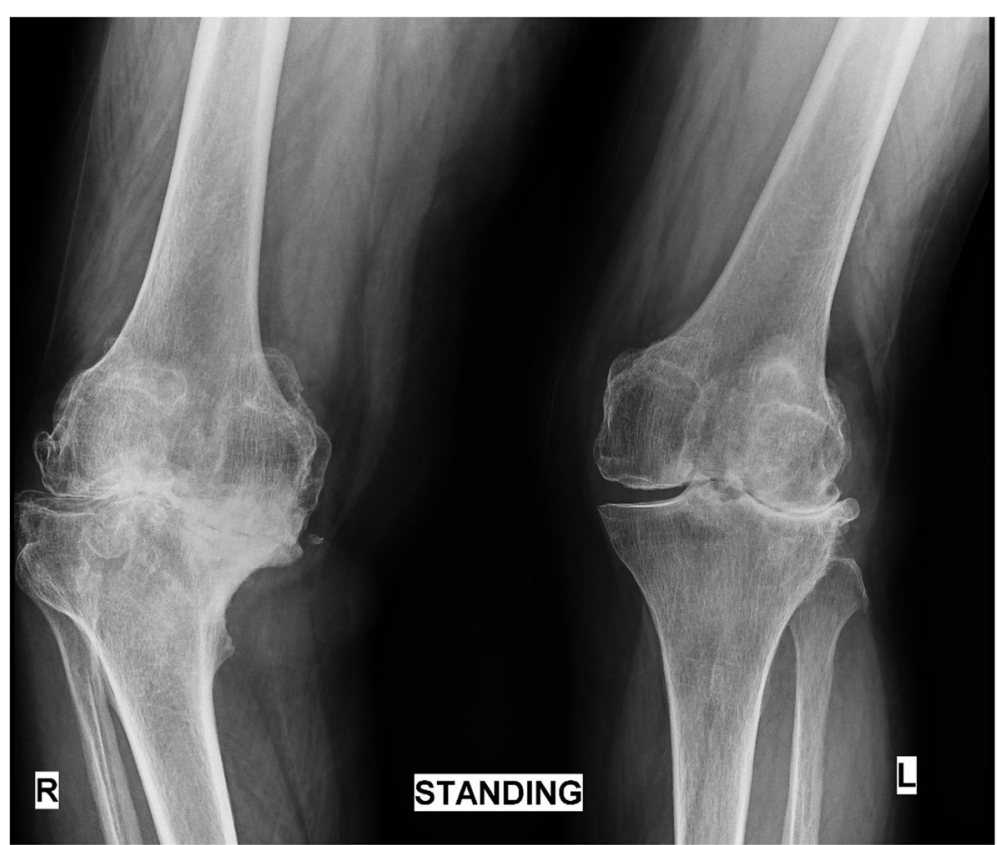

Fig. 3 X-ray of a windswept deformity with combined varus and valgus knees

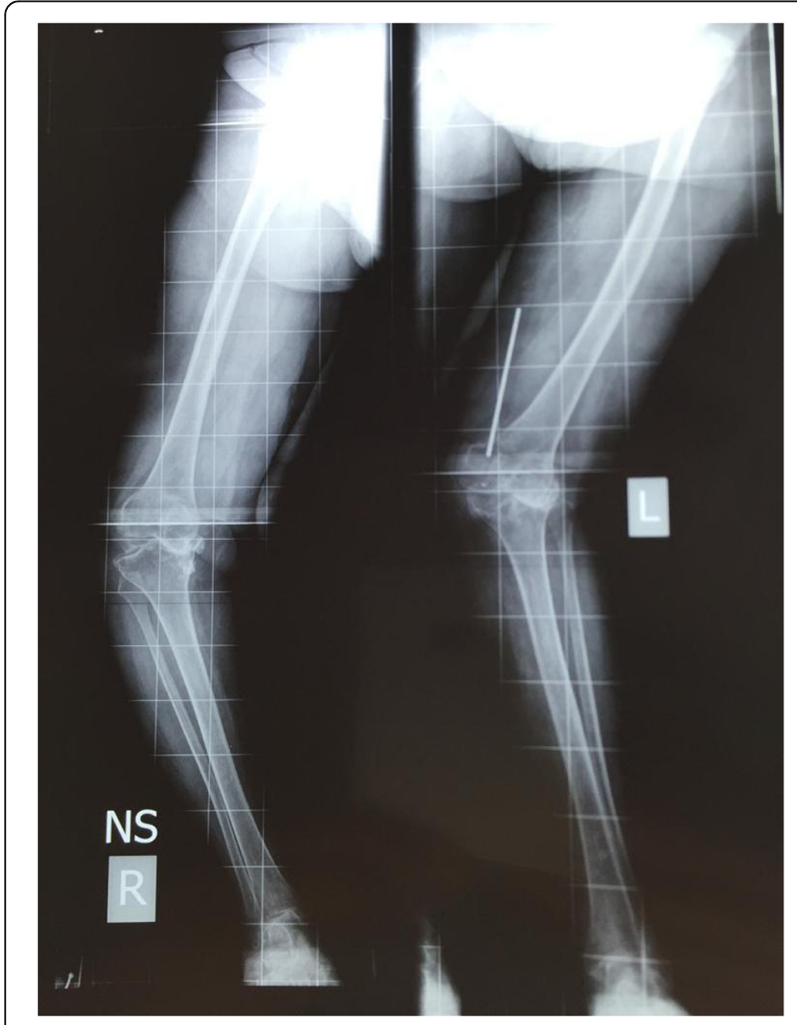

Fig. 4 Scanogram of windswept knees mutually inclusive and need to be addressed as a single problem requiring bilateral TKA $[9,10]$.

\section{Surgical perspective}

We draw from our review of literature and experience that SBTKA in the medically fit group of patients is advantageous for the following reasons:

- It prevents duplication of perioperative procedures and scheduling of operation theatre rosters in a busy set-up [27].

- It avoids the cumulative risk of repeated anesthesia and physiological stress of surgery [28].

- It provides an opportunity to compare the alignment and of both limbs and equalize limb length during surgery while both are sterile-draped [29].

- It provides an opportunity for using autologous bone available from bony resections to build the bony defects [2].

\section{Patient perspective}

Correcting the WSD at a single procedure is a patientfriendly option for the following reasons:

- It is less stressful for the patient [28].

- It avoids the antecedent complications of repeated surgery [28].

- It provides replacement of both knees together (albeit a few months if planned as a staggered procedure) and can be done under a single 
admission and anesthesia, thereby saving on the financial overrun [30].

- An integrated rehabilitation program for both the knees can be followed [30].

- It optimizes the patient-support services in the family and community.

\section{Algorithm}

Based on our experience [31] and the review of the literature, we propose an algorithm for TKA in the management of WSD of the knees (Fig. 5).

\section{Limitations}

This review lacks the robustness due to an absolute paucity of literature that has addressed WSD in totality and as different facets of a single complex deformity. Contemporary literature on TKA in WSD is scarce, and therefore, the results from the limited studies available cannot be adequately validated.

\section{Conclusion}

Knee osteoarthritis with windswept deformities are mutually inclusive and have a compounding effect. The valgus correction angle should be customized to the deformity, and prompt correction of the tibiofemoral angle should be achieved. The deformities in windswept knees are troublesome for the patients and challenging for the surgeons to manage. Outcomes following SBTKA have shown excellent and comparable results to staged procedures and the arthritic knees without WSD. An algorithm for the management of WSD has been presented herewith. A well-planned and judiciously executed SBTKA in the medically fit group of patients offers distinct advantages to the patient and surgeon and provides for optimum utilization of time and resources.

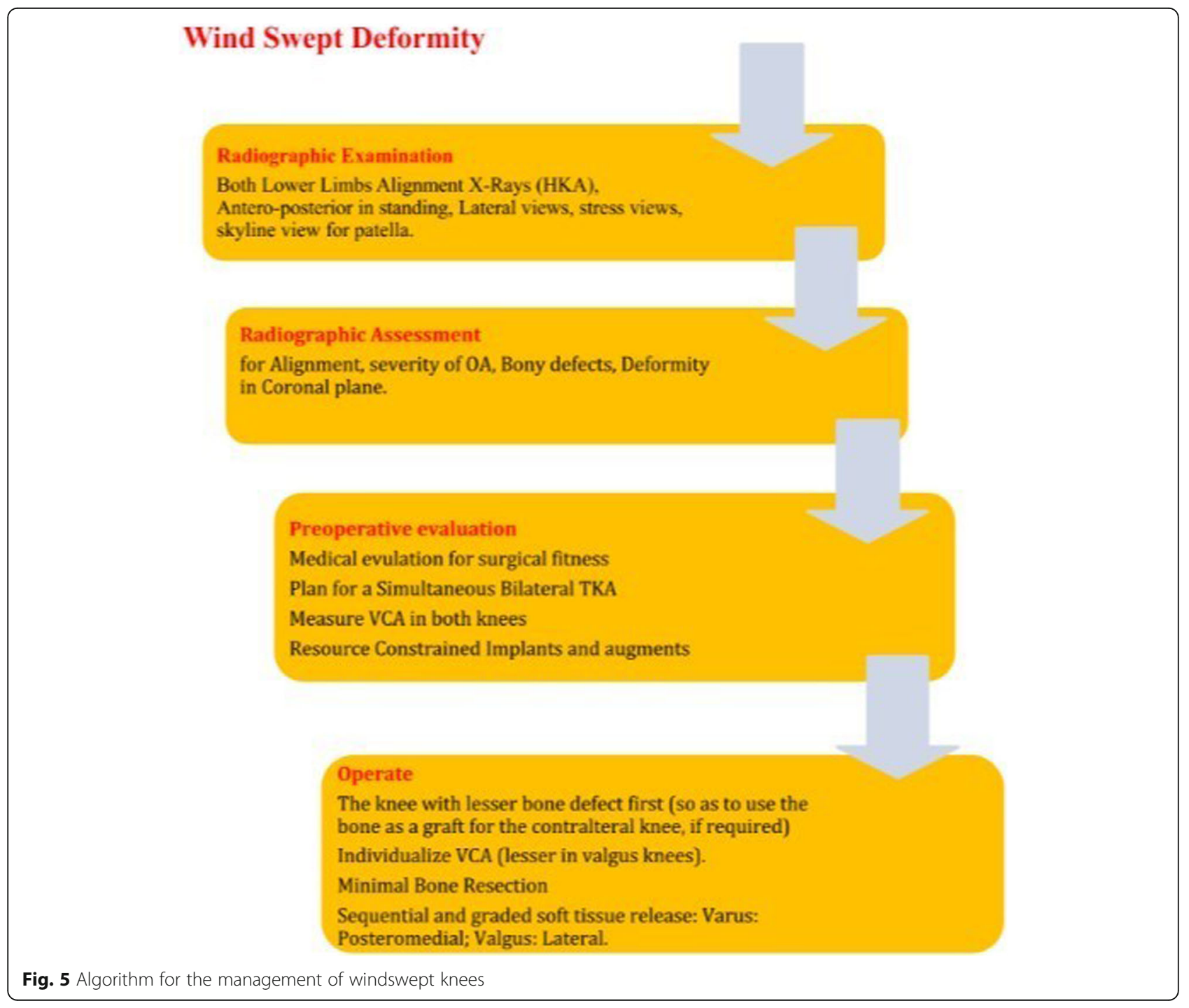




\section{Abbreviations}

CR: Cruciate retaining; HKA: Hip knee ankle; OA: Osteoarthritis; PS: Posterior stabilized; SBTKA: Simultaneous bilateral total knee arthroplasty; TKA: Total knee arthroplasty; VCA: Valgus correction angle; WSD: Windswept deformity

\section{Acknowledgements}

Not applicable.

\section{Authors' contributions}

Suresh Babu was responsible for the review of the literature, analysis, and writing the original manuscript. Abhishek Vaish was responsible for the analysis and the manuscript revision. Raju Vaishya was responsible for the conceptualization and manuscript revision. The authors read and approved the final manuscript.

\section{Funding}

Not applicable.

\section{Availability of data and materials}

As this is a review article, any data quoted in the manuscript was obtained from online databases and is adequately referenced. Table 1 and Fig. 1 are original reproductions; patient photograph and X-rays are sourced from personal archives.

\section{Competing interests}

The authors declare that they have no competing interests.

Received: 11 June 2020 Accepted: 10 August 2020

Published online: 31 August 2020

\section{References}

1. Kim RH, Scott WN. Total Knee Replacement in the Varus Knee. Operative Techniques: Total knee replacement. 2009. p. 91-103.

2. Malhotra R. Chapter-03 Total Knee Arthroplasty in Varus Deformity. Mastering Orthopedic Techniques Total Knee Arthroplasty. 2010. p. 25-42.

3. Catonné Y, Sariali E, Khiami F, Tillie B. Total knee replacement in patients with severe varus deformity. In: Bonnin M, Amendola NA, Bellemans J, MacDonald SJ, Menetrey J, editors. The knee joint: surgical techniques and strategies. Paris: Springer. 2012. p. 915-922.

4. Wright JO, Nunley RM (2015) Complex primary total knee arthroplasty: management of valgus knee. In: Springer BD, Curtin BM (eds) Complex primary and revision total knee arthroplasty: a clinical casebook. Springer, New York, pp 13-27

5. Indelli P, Marcucci M, Faaborg-Andersen C, Poli P, Innocenti M (2015) Correcting varus and valgus knee in total knee arthroplasty (TKA). In: Affatato S (ed) Surgical techniques in total knee arthroplasty and alternative procedures. Elsevier, Amsterdam, pp 151-165

6. Dimitrios N, George S, loannis M (2018) Primary total knee arthroplasty in valgus deformity. In: Zorz AR (ed) Primary total knee arthroplasty. IntechOpen, London

7. Kutzner I, Trepczynski A, Heller MO, Bergmann G (2013) Knee adduction moment and medial contact force - facts about their correlation during gait. PLoS ONE 8(12):e81036

8. Amin S, Luepongsak N, McGibbon CA, Lavalley MP, Krebs DE, Felson DT (2004) Knee adduction moment and development of chronic knee pain in elders. Arthritis Care Res 51(3):371-376

9. Andriacchi TP, Koo S, Scanlan SF (2009) Gait mechanics influence healthy cartilage morphology and osteoarthritis of the knee. J Bone Joint Surg Am 91(Suppl 1):95-101

10. Mündermann A, Dyrby CO, Andriacchi TP (2005) Secondary gait changes in patients with medial compartment knee osteoarthritis: increased load at the ankle, knee, and hip during walking. Arthritis Rheum 52(9):2835-2844

11. Beveridge J, Sharma G, Kuntze G, Jaremko J, Koles S, Wiley J et al (2015) A preliminary study of contralateral knee structure and function associations in persons with unilateral knee osteoarthritis. Osteoarthr Cartil 23:A124

12. Kaufman KR, Hughes C, Morrey BF, Morrey M, An K-N (2001) Gait characteristics of patients with knee osteoarthritis. J Biomech 34(7):907-915

13. Arizono T, Ogata K, Sugioka Y (1989-1990) Windswept deformity of the knee in adult. Orthop Traumatol-Sur 38:1703-1170

14. Case JP, Shakoor Baliunas AJ, Block JA, Hurwitz DE. Unilateral knee osteoarthritis, the relative overloading of the contralateral noncognate joints and the relationship to pain. Division of Rheumatology, Cook County Hospital, Chicago, IL. 49th Annual Meeting of the Orthopaedic Research Society. 2003.

15. Rao MK, Reddy PLN (2017) Frontal plane tibiofemoral alignment and its association with knee pain among patients with early osteoarthritis of the knee in a population presenting to a tertiary hospital in South India. Int J Res Orthop 4(1):79

16. Sharma $L$ (2007) The role of varus and valgus alignment in knee osteoarthritis. Arthritis Rheum 56(4):1044-1047

17. Shetty GM, Mullaji A, Khalifa AA, Ray A (2017) Windswept deformities - an indication to individualize valgus correction angle during total knee arthroplasty. J Orthop 14(1):70-72

18. Shi X, Li H, Zhou Z, Shen B, Yang J, Kang P et al (2015) Individual valgus correction angle improves the accuracy of postoperative limb alignment restoration after total knee arthroplasty. Knee Surg Sports Traumatol Arthrosc 25(1):277-283

19. Nam D, Vajapey S, Haynes JA, Barrack RL, Nunley RM (2016) Does use of a variable distal femur resection angle improve radiographic alignment in primary total knee arthroplasty? J Arthroplast 31(9 Suppl):91-96. https://doi. org/10.1016/j.arth.2016.01.070.

20. Zhou K, Zhou Z, Shi X et al (2018) Effect of individual distal femoral valgus resection in total knee arthroplasty for patients with a valgus knee: a retrospective cohort study. Int J Surg 52:309-313. https://doi.org/10.1016/j. ijsu.2018.02.048.

21. Howell SM, Shelton TJ, Gill M, Hull ML (2020) A cruciate-retaining implant can treat both knees of most windswept deformities when performed with calipered kinematically aligned TKA. Knee Surg Sports Traumatol Arthrosc. https://doi.org/10.1007/s00167-020-05968-9

22. In-soo Song MD, Je-gyun Chon MD, Jun-beom Kim MD (2008) Total knee arthroplasty for treating valgus and varus in the knees of one person. J Korean Knee Soc 20(2):110-116

23. Baldini A, Balato G, Franceschini V (2015) The role of offset stems in revision knee arthroplasty. Curr Rev Musculoskelet Med 8(4):383-389

24. Meding JB, Anderson AR, Ritter MA, Faris PM, Keating E (2000) Windswept deformity in bilateral total knee arthroplasty. J Arthroplast 15(5):562-566

25. Kentaro H, Yoshihiro M (2004) Total knee arthroplasty for windswept deformity-a case report. Kawasaki Med Sch 30(3-4):179-182. Available from: http://igakkai.kms-igakkai.com/wp/wp-content/uploads/2004/KMJ30(3,4)1 79-182.2004.pdf.

26. Al KA, Ganger R, Klaushofer K, Grill F (2012) Windswept deformity in a patient with Schwartz-Jampel syndrome. Swiss Med Wkly 19(1112):142

27. Turrentine FE, Wang H, Simpson VB, Jones RS (2006) Surgical risk factors, morbidity, and mortality in elderly patients. J Am Coll Surg 203(6):865-877. https://doi.org/10.1016/j.jamcollsurg.2006.08.026 PMID: 17116555

28. Story DA (2008) Postoperative complications in elderly patients and their significance for long-term prognosis. Curr Opin Anaesthesiol 21(3):375-379. https://doi.org/10.1097/ACO.0b013e3282f889f8.

29. Bagsby D, Pierson JL (2015) Functional outcomes of simultaneous bilatera versus unilateral total knee arthroplasty. Orthopedics 38:e43-e47. https://doi. org/10.3928/01477447-20150105-59.

30. March L, Cross M, Tribe K, Lapsley H, Courtenay B, Cross M et al (2004) Two knees or not two knees? Osteoarthr Cartil 12(5):400-408

31. Vaishya R, Vijay V, KC KM, Agarwal AK (2018) Is simultaneous bilateral total knee arthroplasty safe in the geriatric population? A retrospective cohort study with up to 9 years follow up. J Clin Orthop Trauma 9(2):107-111

\section{Publisher's Note}

Springer Nature remains neutral with regard to jurisdictional claims in published maps and institutional affiliations. 